\author{
Magdalena Nigoevic ${ }^{*}$ \\ Sonja Carić** \\ Università degli Studi di Spalato
}

\title{
L'ETICHETTAMENTO DEGLI IMMIGRATI NELLA STAMPA ITALIANA
}

\begin{abstract}
Questo studio affronta la rappresentazione degli immigrati nei quotidiani italiani, più precisamente negli articoli di cronaca nera. L'etichettamento degli immigrati nei giornali italiani non consiste solo nello scegliere delle informazioni, vale a dire in una pubblicazione sistematica di notizie negative. Esso è evidente soprattutto al livello delle scelte linguistiche, rispettivamente attraverso l'uso costante di un linguaggio che favorisce una percezione stereotipata, spesso discriminatoria.

Si analizzano i principali bias linguistici che contribuiscono alla formazione di una diffusa opinione negativa sugli immigrati. Il corpus è stato raccolto attraverso la lettura sistematica di articoli pubblicati nei quotidiani nazionali. Nel corso dell'analisi, si è potuto osservare che esiste una tendenza ad enfatizzare la nazionalità degli immigrati, in modo quantitativo - ripetendolo più volte nel corpo dell'articolo, oppure in modo qualitativo - posizionandolo in punti importanti, nei titoli. Inoltre, si può constatare che il numero di altri determinanti, di qualsiasi genere, è basso rispetto al numero di etnici usati, sia nella forma aggettivale o in quella sostantivata.

Parole chiave: etichettamento, immigrati, linguaggio discriminatorio, quotidiani italiani, cronaca nera.
\end{abstract}

\section{INTRODUZIONE}

Esiste un passaggio nella costruzione di una identità deviante, "costituito dal meccanismo attraverso il quale un soggetto che ha messo in atto un comportamento deviante viene identificato come deviante ed etichettato come tale con l'applicazione di una label (etichetta) ${ }^{1}$ che non copre solo il comportamento specifico identificato come deviante, ma si allarga a tutta

*magda@ffst.hr

***soncar@ffst.hr

${ }^{1} \mathrm{Si}$ fa riferimento alla teoria dell'etichettamento (Labelling Theory) nata negli anni Sessanta del secolo scorso negli Stati Uniti ed elaborata da Becker (1963). È una teoria sociologica che vede la devianza come una costruzione sociale. Si focalizza sul "processo" del divenire deviante, in cui giocano un ruolo fondamentale i processi di attribuzione, di etichettamento e di stigmatizzazione che colpiscono la condotta deviante. 
l'identità sociale del soggetto" (Solivetti 2004: 36). Il presente studio intende affrontare il tema dell'etichettamento linguistico usato dai giornali italiani. L'importanza di questa forma di etichettatura, che è spesso discriminatoria, risiede nel fatto che la lingua e l'ambito linguistico non sono soltanto "uno strumento di comunicazione attraverso il quale vengono trasmesse informazioni e idee. Essa [lingua] riflette nei suoi usi la società che la utilizza. Nello stesso tempo, però, la lingua influenza la società nel modo di pensare, di giudicare, di classificare la realtà." (Fioritto 1997: 37).

Il termine stesso 'discriminazione' include la nozione di disparità, specialmente di trattamento, cioè "la violenza o l'odio nei confronti di gruppi di persone" (MDEND 2011: 111) definiti in base ad un certo tratto, che può essere la razza, ''etnia $^{2}$, la religione, l'ascendenza, l'orientamento sessuale, o qualsiasi altro criterio. Una delle caratteristiche fondamentali di tutti questi termini è che, anche se ben distinguibili dal punto di vista linguistico, nella percezione umana vengono spesso considerati interdipendenti, connessi anche con l'origine sociale, la nascita e la ricchezza e, in quanto tali, sono tutti criteri discriminatori.

\section{MEDIA E IMMIGRATI}

Secondo il RFC (2002), un'immagine degli immigrati, il cosiddetto 'immigrato ${ }^{3}$ tipo', che si può trarre dai media, in questo caso dalla televisione, è un'immagine maschile, non oltre i 65 anni e per lo più appartenente alla cronaca nera ${ }^{4}$ del dato giorno. Secondo i ricercatori, gli argomenti affrontati in televisione riguardo agli immigrati e/o stranieri sono, in più del $50 \%$ dei casi, la criminalità e l'illegalità (cfr. RFC 2002: 14). Non è, comunque, un fenomeno esclusivamente italiano. Latrofa e Vaes (2013: 19) riportano i risultati di studi che documentano la discriminazione dei media verso gli afroamericani, rappresentati come criminali in misura maggiore della reale incidenza (2013: 19). Un altro caso di discriminazione linguistica è stato

${ }^{2}$ Anche se questi due termini vengono spesso usati in modo intercambiabile, ci sono delle differenze. Il primo, razza, si riferisce alla suddivisione di una specie "in base a caratteri somatici quali il colore della pelle, la forma degli occhi o del cranio, la statura media, ecc." (Zingarelli 2012: 1876), in altre parole, si tratta di una classificazione biologica, in base alle caratteristiche morfologiche. Il termine etnia, invece, si definisce come "raggruppamento umano basato su comuni caratteri fisico-somatici, linguistici e culturali" (Zingarelli 2012: 837), cioè, l'enfasi viene data ai contesti culturali e/o tradizionali dai quali dipende l'accomunamento dei gruppi sociali.

${ }^{3}$ Il concetto di immigrato non è privo della dualità tra quanto percepito come nostro e quanto percepito come straniero perché si tratta di colui che "si è stabilito in un paese straniero o in un'altra regione della propria nazione" (Zingarelli 2012: 1075).

4 "In oltre l' $80 \%$ dei casi si tratta di uomini, e in meno del $20 \%$ di donne."; "il 43,5\% è cronaca nera e [...] il 2,4\% giudiziaria" (cfr. RFC 2002: 7, 9). 
documentato in Australia, dove l'uso dei termini Aboriginal, Koori e Australian variava secondo il tipo, cioè il contenuto positivo o negativo della notizia (cfr. WYL 2005: 20) .

Latrofa e Vaes (2013: 18) sostengono che "gli atteggiamenti nei confronti di alcune categorie sociali, quali gli omosessuali, i musulmani, gli immigrati, possano essere influenzati da un'elevata esposizione a rappresentazioni negative e stereotipate diffuse dai mezzi di comunicazione di massa". Infatti, i media costruiscono una rappresentazione della realtà sociale "che viene, poi, generalmente, adottata da chi ascolta (e/o legge) come "la" rappresentazione, oggettiva ed incontrovertibile" (Fiorini 2003). La discriminazione degli immigrati nei giornali italiani non consiste solo nello scegliere delle informazioni, vale a dire in una pubblicazione sistematica di notizie negative - la discriminazione si ha anche al livello dell'uso della lingua. Attraverso l'uso costante di un linguaggio che favorisce una percezione negativa, "prima ancora di essere discriminati nei fatti, migranti e profughi sono discriminati dal linguaggio che la nostra società escogita per rappresentarli” (Dal Lago 2009: 43).

\section{ETICHETTAMENTO LINGUISTICO}

Tenendo in considerazione le ricerche precedenti simili (Mahmoud 1990, Corte 2002, Dal Lago 2009) abbiamo cercato di individuare alcune tendenze linguistiche discriminatorie. Si è dedicata una specifica attenzione ai principali bias linguistici proposti da $\mathrm{Vaes}^{6}$ che contribuiscono alla formazione di una diffusa opinione negativa verso gli immigrati. Questi sono: riferimenti alla nazionalità, group-first, omissione del nome proprio, uso di aggettivi negativi, diatesi verbale e linguaggio metaforico. I riferimenti alla nazionalità comprendono l'uso di aggettivi etnici, aggettivi che denominano gli abitanti e/o i provenienti da una certa nazione o da qualsiasi altra area geografica. Nei giornali italiani, è anche "frequente che il richiamo alla provenienza non italiana dell'autore del reato compaia sotto forma di agget-

${ }^{5}$ Come sottolineato nello stesso testo, in Australia è anche illegale specificare dettagli tipo "gender, race, age, marital, parental or career status, sexual orientation" (WYL 2005: 18), almeno in ambito professionale.

${ }^{6}$ Jeroen Vaes del Dipartimento di Psicologia dello sviluppo e della socializzazione dell'Università di Padova è coordinatore del progetto "Immigrazione, paura del crimine e i mass media: ruoli e responsabilità", affidatogli dalla Fondazione CARIPARO nel 2009. Preso da: http://www.mmc2000.net/mediattivi/immigrazione-paura-del-crimine-e-i-mediail-linguaggio-discriminatorio-della-stampa/ (23/12/2013). 
tivo sostantivato piuttosto che di semplice aggettivo", riducendo così una persona ad un singolo tratto, la nazionalità ${ }^{8}$. Il cosiddetto group-first è una forma di stereotipizzazione ${ }^{9}$ che anticipa le informazioni sull'appartenenza sociale a spese delle caratteristiche individuali della persona coinvolta. Questa tecnica è strettamente connessa all'uso di aggettivi negativi (ing. negative labelling) e all'uso di forme verbali attive. Mediante questo uso si comunica l'idea di un livello di colpevolezza molto più alto rispetto a quello dei criminali italiani.

\section{METODOLOGIA}

A causa della grande importanza dei mezzi di comunicazione e della loro capacità di influenzare le percezioni della realtà da parte dei lettori di massa, si è scelto di analizzare i modi di etichettamento linguistico presenti negli articoli di cronaca nera.

L'analisi comprende gli esempi tratti dagli articoli apparsi nelle edizioni elettroniche dei giornali Corriere della Sera e La Repubblica. Il corpus è stato raccolto attraverso la lettura sistematica di articoli pubblicati durante il periodo di un mese, dal 1 novembre al 30 novembre del 2013. Gli articoli dai quali sono stati citati gli esempi, appartengono alla cronaca nera che comprende gli avvenimenti solitamente correlati a reati e operazioni poliziesche che hanno come scopo il mantenimento della normale convivenza sociale. Registrano di solito avvenimenti quali risse, furti, incidenti, omicidi, rapine ecc. Il materiale dal quale è stato raccolto il corpus contiene settantotto (78) articoli. In seguito, gli esempi estratti vengono classificati e commentati per osservare le caratteristiche dell'uso dei riferimenti alla nazionalità nel linguaggio giornalistico ed il loro impatto sul modo in cui vengono percepiti gli immigrati stessi.

\section{CLASSIFICAZIONE ED ANALISI DEGLI ESEMPI}

Tutti gli articoli presi in considerazione contengono un riferimento diretto alla nazionalità della persona straniera coinvolta, sia nel ruolo di

${ }^{7}$ Ibid.

8 “"...] l'uso così diffuso del richiamo alla nazionalità rappresenta la tendenza a ricondurre l'immigrato alla sua dimensione di straniero, ricollocandolo nel suo Paese di provenienza" (RFC 2002: 13).

9 "Stereotyping describes an individual only in group terms rather than as someone with a distinct identity. When individuals or groups are labelled in stereotypical ways, they may experience hurt and pain, contributing to a negative self image and feelings of inferiority" (WYL 2005: 5). 
aggressore, che nella posizione di vittima. Nel corso di quest'analisi, gli articoli $\mathrm{o}$, per meglio dire, $\mathrm{i}$ riferimenti alla nazionalità saranno suddivisi $\mathrm{e}$ commentati attraverso le seguenti categorie:

A. nazionalità indicata nel titolo

B. nazionalità indicata nel corpo dell'articolo

C. l'uso della costruzione "di origine X"

D. l'uso di etnici insieme ad altri termini descrittivi

E. l'indicazione della nazionalità negli articoli in cui gli immigrati sono le vittime.

A. La prima categoria, comprendente gli articoli in cui il riferimento alla nazionalità si fa nel titolo, consiste di 24 articoli, ovvero il 30,7\% del numero totale. Alcuni degli esempi sono:

(1) Accoltellata in casa: Ecuadoriano ${ }^{10}$ ricercato (CS 03/11/2013). ${ }^{11}$

(2) Abusi sulla figlia dell'ex compagna: Romeno in cella (CS 06/11/2013).

(3) Noviglio, banda di ladri albanesi tradita dall'auto di lusso: arrestati (CS 12/11/2013).

(4) Uccide il cugino: arrestato egiziano (CS 17/11/2013).

(5) Sette banditi serbi in fuga inseguiti e fermati al casello (CS 20/11/2013).

(6) Ragazza americana denuncia "Io, stuprata da sei stranieri" (CS 22/11/2013).

(7) Massacrata in casa, fermato 18enne nigeriano (LR 14/11/2013).

(8) Rapine nelle ville arrestati sette slavi (LR 20/11/2013).

In primo luogo bisogna sottolineare che la menzione della nazionalità non è limitata soltanto al titolo. Invece, la nazionalità del delinquente si ripete più volte nel corpo dell'articolo, anche quando vengono specificate altre caratteristiche del criminale (il nome o, più di frequente, l'età). Un altro fattore degno di nota è che viene riportata anche la nazionalità della vittima, specialmente se la vittima in questione non è di nazionalità italiana. Non mancano, in questi casi, gli usi di termini tipo 'connazionale': "Una donna ecuadoriana di 35 anni è stata ferita a coltellate l'altra notte in un appartamento di via Padova. A colpire, un connazionale di 38 anni che l'avrebbe sorpresa durante il sonno" (CS 03/11/2013). Questa parola viene adoperata nei casi in cui c'è più di un coinvolto: "Il marocchino e un suo connazionale si infilano dentro, col volto semicoperto da un cappuccio" (CS

${ }^{10} \mathrm{Il}$ corsivo è nostro.

${ }^{11}$ Dopo le citazioni si adoperano le sigle CS per Il Corriere della Sera e LR per La Repubblica. 
22/11/2013). L'ormai enfatizzata origine dei coinvolti è posta ulteriormente in rilievo mediante parole come 'clandestino', 'immigrato' 12 e simili.

L'esempio (6) considera il titolo di un articolo su una ragazza stuprata a Roma da sei ragazzi che denota una disparità di trattamento fra nazionalità della vittima e degli aggressori. Il titolo infatti riporta le parole della ragazza ${ }^{13}$ qualificata come 'americana' rispetto a dei ragazzi 'stranieri', evidenziando una presunta superiorità della vittima rispetto agli agressori. Inoltre, la seguente citazione dell'articolo "la giovane, da tempo a Roma dove lavora presso un istituto di studi linguistici al Flaminio" (CS 22/11/2013) evidenzia la tendenza ad "italianizzare" socialmente la vittima. In particolare si può riscontrare che la nazionalità è definita in modo approssimativo perché i sei ragazzi che hanno stuprato la ragazza americana sono definiti "sei giovani dall'aspetto mediorientale" (CS 22/11/2013). Facendo così, il group-first negli articoli si estende anche oltre i limiti della nazione dei criminali, ad un termine ancora più generico ${ }^{14}$.

B. Questa categoria comprende 25 articoli (il 32\% del numero totale) che indicano l'origine nel corpo del testo. Alcuni degli esempi sono:

(9) "Vittima una studentessa di 19 anni, rapinata mercoledì sera da un nordafricano" (CS 08/11/2013).

(10) "I carabinieri di Vigevano l'hanno liberata e arrestato gli autori del rapimento: cinque suoi connazionali, tutti rumeni" (CS 14/11/2013).

(11) "Attimi drammatici in piazza Verbano, al Salario, dove cinque sudamericani, fra i quali una donna, sono fuggiti senza lasciare traccia con alcune decine di euro" (CS 28/11/2013).

(12) "Una lama di dieci centimetri nella schiena della sorella: arrestata per tentato omicidio a Viterbo una ragazza di 28 anni dell'est Europa" (LR 09/11/2013).

Siccome negli articoli si descrive il delitto commesso, bisogna prestare attenzione alla diatesi attiva e passiva ivi presente. Si usano ambedue le costruzioni, quella attiva e quella passiva, tuttavia si può notare una leggera differenza. Più precisamente, si è osservato che l'attivo prevale nei casi dove esiste una relazione tra l'aggressore e la vittima, sia familiare che affettiva,

${ }^{12} \mathrm{Si}$ veda: "Un immigrato marocchino di 22 anni, irregolare in Italia, è stato arrestato lunedì dalla polizia ferroviaria della stazione centrale di Milano" (CS 13/11/2013)

${ }^{13}$ Anche se la frase "Io, stuprata da sei stranieri" (CS 22/11/2013) è stata messa tra virgolette, non si sa se queste siano state le parole della ragazza, riportate parola per parola, o se si tratti di un esempio di licentia giornalistica.

${ }^{14}$ Ciò vale anche per l'esempio (8) dove sono stati “arrestati sette slavi” (LR 20/11/2013). 
in altre parole di conoscenza ${ }^{15}$. Riguardo alla marcatura della nazionalità, in un numero considerevole di articoli, spesso c'è una tendenza ad evitare l'accentuazione dell'origine degli attaccanti, rilevando perciò l'origine delle vittime. Tra gli esempi, se ne riporta uno: "La donna originaria dello Sri Lanka aveva avuto una relazione col suo aggressore, un connazionale di 42 anni" (CS 20/11/2013).

Una particolarità assai importante si nota in questi due esempi: "Aggrediti da due tunisini con un coltello che volevano la maglia della Nazionale di Mario Balotelli indossata da una delle vittime" (CS 07/11/2013) e "L'indagato [N.d.A.: un marocchino], riconosciuto dalle due donne anche grazie alla pettinatura ispirata a Balotelli, ha solo 15 anni, ma la forza e l'animo violento di un uomo consumato e brutale" (CS 23/11/2013). L'uso, nel secondo esempio, del cognome di Mario Balotelli, il famoso calciatore italiano, può essere interpretato come un'allusione suggestiva all'origine dell'atleta, che è in realtà figlio di immigrati ghanesi. Il fatto che col suo cognome vengono connessi delinquenti provenienti da due stati africani, Tunisia e Marocco si può spiegare come negli esempi precedenti sugli aggressori slavi e mediorientali. Gli stati economicamente deboli sono di solito meno riconosciuti. Infatti, si riscontra con maggiore frequenza l'espressione 'nordafricano' per indicare per esempio 'un rapinatore marocchino', come nell'esempio (9), rispetto all'espressione 'nordeuropeo' per descrivere 'un criminale finlandese'.

C. Legata alla precedente è la categoria di articoli analizzati in cui gli immigrati sono descritti come persone "di origine X". Sono sette (7) i testi in questa categoria, tra cui:

(13) "Ex operai di origine slava, amici di 31 e 34 anni, per tirare alla fine del mese, si sono improvvisati criminali" (CS 07/11/2013).

(14) "Il 15enne, di origini magrebine, fermato venerdì scorso con l'accusa di aver violentato due donne, respinge tutte le accuse" (CS 26/11/2013).

(15) "Un uomo di origine moldava, A.T., di 48 anni, è stato arrestato dalla Polizia a Remanzacco (Udine) dopo aver ucciso a coltellate il figlio di 19 anni e ferito in modo grave la moglie, ricoverata in rianimazione all'ospedale di Udine" (LR 26/11/2013).

Sull'uso di questo tipo di marcature non si possono dare spiegazioni esatte ed accertate. Al massimo, si può soltanto constatare che con l'uso della

${ }^{15}$ Alcuni degli esempi: "“Sono Allah'. Aggredisce moglie e figlia“ (CS 30/11/2013), "Sequestra e stupra la ex che non lo sposa: arrestato" (LR 20/11/2013) e "Uccide il cugino durante una lite: venticinquenne arrestato a Monza" (LR 16/11/2013). 
costruzione "di origine X" si ottiene una minore esclusione e distanziamento dei criminali immigrati. Oltre a ciò, in tutti e tre gli esempi elencati esiste una circostanza attenuante: nell'esempio (13) questa è la disoccupazione dei due slavi, in (14) vi sono l'età e l'assenza di una confessione da parte del quindicenne e, infine, nell'esempio (15) l'ubriachezza dell'uomo.

D. Nei dodici (12) articoli di questa categoria i riferimenti etnici sono uniti ad altri elementi descrittivi, come si vede dai seguenti esempi:

(16) 'PALERMO: LA SOCIETÀ DELL'EX ATLETA UCRAINA FORNIVA BARCHE DI LUSSO. COINVOLTE SETTE PERSONE" (CS 01/11/2013).

(17) "Succede tutto verso le due dell'altra notte quando $\mathrm{Nelu} \mathrm{Ca}$ sangiu, camionista romeno di 40 anni, decide di fare inversione all'improvviso e va a schiantarsi con un'altra vettura" (CS $02 / 11 / 2013)$.

(18) “A ventiquattro anni d'età, il somalo Elmi Mouhamud Muhidin faceva il predatore di migranti” (LR 09/11/2013).

Come si osserva dagli esempi, i determinanti più usati sono il mestiere come 'l'ex atleta' nell' esempio (16), oppure il mestiere accompagnato con il nome e cognome del criminale come in (17). Questo può essere un segno di riconoscimento (il nome dell'atleta può essere noto agli amanti dello sport), un fattore rilevante al reato (camionista che guida ubriaco), oppure la personalizzazione di un aggressore particolarmente crudele in (18).

L'esempio molto singolare è il caso di Behexhed Bushi, un'albanese su cui il Corriere della Sera ha pubblicato vari articoli. È interessante seguire, attraverso questo caso, il processo di progressiva personalizzazione: negli ultimi articoli pubblicati, lui è “l'albanese" (CS 27/11/2013) con l'articolo determinativo, mentre nel titolo dell'ultimo si legge "I familiari di Bushi" (CS 28/11/2013). Con una serie di articoli che considerano lo stesso personaggio si raggiunge un certo livello di conoscenza nei lettori del giornale e tale avvicinamento è evidente anche attraverso le scelte di denominazione.

E. Infine, undici (11) articoli analizzati hanno come vittime le persone d'origine straniera:

(19) "A perdere la vita ieri nel pomeriggio è stato Dinu Balazs Tinis, un romeno di 34 anni residente a Lodi, colpito alle spalle dal fucile dell'amico mentre era alla guida della sua Ford Galaxy" (CS 25/11/2013). 
(20) "Una studentessa cinese di 24 anni è stata aggredita e rapinata della borsa mentre rientrava a casa l'altra notte in via Chopin" (CS 02/11/2013).

L'unica caratteristica che li accomuna è la loro relativa brevità e concisione nel riportare l'accaduto. Rispetto agli articoli in cui gli immigrati sono gli aggressori, la quantità dei dettagli riportati è ridotta alla sola descrizione del reato commesso, presentato con uno stile leggero.

\section{CONCLUSIONE}

Lo scopo dell'analisi condotta era esaminare il linguaggio usato negli articoli di cronaca nera che trattano i crimini commessi o subiti dagli immigrati. Nel corso dell'analisi, si è giunti alla conclusione che c'è una tendenza ad enfatizzare la nazionalità degli immigrati, in modo quantitativo, cioè ripetendolo più volte nell'articolo, $\mathrm{o}$ in modo qualitativo, posizionandolo in luoghi di importanza, cioè nei titoli. Il numero di altri determinanti, di qualsiasi genere (il nome, l'occupazione e sim.) è basso rispetto al numero di etnici usati, sia nella forma aggettivale che in quella sostantivata. Inoltre, si potrebbe notare che l'uso del sintagma preposizionale "di origine X" è più comune nei casi dove esistono delle circostanze attenuanti, mentre non sono tanti i casi in cui gli immigrati sono le vittime e per lo più sono esposti in maniera "telegrafica".

Indubbiamente queste scelte linguistiche possono contribuire ad un processo di generalizzazione, in cui i lettori sono indotti ad aspettarsi un livello alto di criminalità in tutti gli immigrati che, infine, potrebbe risultare in un maggiore livello di discriminazione. Marcando la nazionalità e l'appartenenza etnica della persona in questione, aumenta anche il numero percepito dei crimini che coinvolgono gli immigrati. Per cambiare ed eliminare queste etichettature linguistiche, non basta adottare delle nuove modalità espressive: ci vorrebbe un'analisi delle convenzioni e delle abitudini nel parlare e nello scrivere, con lo scopo di distinguere e classificare le supposizioni e i valori che stanno dietro alle etichettature linguistiche.

\section{BIBLIOGRAFIA}

Becker, H.S. (1963). Outsiders: Studies in the Sociology of Deviance. New York: The Free Press (tr. it. Outsiders. Saggi di sociologia della devianza. Torino: Edizioni Gruppo Abele, 1987).

Corte, M. (2002). Noi e gli altri: L'immagine dell'immigrazione e degli immigrati sui mass media Italiani. Verona: CESTIM - Centro Studi Immigrazione Onlus. 
Dal Lago, A. (2009). Non persone. L'esclusione dei migranti in una società globale. Milano: Feltrinelli.

Fiorini, A. (2003). Immigrazione e mass media. Disponibile all'indirizzo: http://www.sestaopera.it/DOCUMENTI/DOSSIER/Fiorini_Immigrazione.pdf (consultato 17 gennaio 2014).

Fioritto, A. (a cura di). (1997). Manuale di stile: Strumenti per semplificare il linguaggio delle amministrazioni pubbliche. Bologna: Il Mulino.

Latrofa, M. e Vaes, J. (2013). Potere mediatico e pregiudizio: I mass-media influenzano la nostra percezione sociale? The Inquisitive Mind Italia, $3,18-24$.

Mahmoud, M. (1990). Noi, stranieri d'Italia. Immigrazione e mass-media. Lucca: Maria Pacini Fazzi Editore.

MDEND (2011). Manuale di diritto europeo della non discriminazione. Lussemburgo: Ufficio delle pubblicazioni dell'Unione europea.

RFC (2002). Rapporto finale della Fondazione Censis. Roma: Fondazione Censis.

Solivetti, L. M. (2004). Immigrazione, integrazione e crimine in Europa. Bologna: Il Mulino.

WYL (2005). Watch Your Language: Guidelines for Non-discriminatory Language. Melbourne: Equal Opportunity Unit, University of Melbourne. Testo disponibile al sito: https://hr.unimelb.edu.au/ data/assets/pdf_file/0003/87501/Watch_Your_Language.pdf (consultato 17 gennaio 2014).

Zingarelli, N. (2012). Vocabolario della lingua italiana. Bologna: Zanichelli.

\section{Sitografia}

http://www.mmc2000.net/mediattivi/immigrazione-paura-del-crimine-ei-media-il-linguaggio-discriminatorio-della-stampa/ (consultato 23 dicembre 2013). 


\section{LABELLING OF IMMIGRANTS IN ITALIAN NEWSPAPERS}

\section{Summary}

This study deals with the representation of immigrants in Italian daily newspapers or, more precisely, crime news. The labelling of immigrants in Italian newspapers consists not only of the selection of information, which comprises a systematic publication of negative information, but is also evident in linguistic choices. In other words, it is possible to discern a constant usage of such language which favors a stereotyped, often discriminatory perception.

The principal linguistic biases which contribute to the formation of a pervasive negative attitude towards immigrants are analyzed. The corpus has been collected by a systematic reading of articles published in daily newspapers. In the course of the analysis, it was noted that the tendency towards an overt emphasis of an immigrant's nationality exists in two forms: quantitative - by repeating it throughout the article, and qualitative - by placing these lexical items in positions of importance, such as headlines. Furthermore, it is ascertained that the number of other markers, of any sort, is low when compared to the number of ethnicity markers used in the articles, both in adjectival and noun form.

Keywords: labelling, immigrants, discriminatory language, Italian newspapers, crime news. 

Segnalazioni 
